\title{
An Idea to a World inside a Black Hole
}

\author{
T. G.M. Gerlitz*, W. Walden \\ Department of Computer Sciences, Technological University of Panama, Republic of Panama \\ *Corresponding author: gerlitz@yahoo.com
}

\begin{abstract}
An investigation on black holes reveals interesting new data. Due to the many speculations about the inside of black holes a concept is developed to allow consideration of the properties occuring inside on a model consisting of an electromagnetic wave. The advantage of the current model is that the investigation is based on a mass originated from even that wave and rather avoids to base the study on an already existing massive mass, which then preliminary excludes any transparent imagination in that field. The theory starts on a classical treatment to later incorporate relativistic considerations leading finally to the transformation equations appropriate for a description of an anti-world. On the basis of the photo sphere the Schwarzschild radius can be determined, which is completely free and independent then on any preliminary given conditions those could ban further in-depth treatment of that task. It is shown each of the two frames reveals individually its own Schwarzschild radius, both of them being reciprocal to each other. In spite of their distinct characteristics of the frames inside and outside a black hole it can be stated out those radii touch each other. A comparison of the two frames can be established by a set of transformation equations to justify the physical properties of the two frames are ideally mirrored in accordance to CPT-operation. The possibility of an anti-universe inside a black hole is discussed.
\end{abstract}

Keywords: Charge conjugation, parity, time reversal

Cite This Article: T. G.M. Gerlitz, and W. Walden, "An Idea to a World inside a Black Hole." International Journal of Physics, vol. 5, no. 5 (2017): 171-180. doi: 10.12691/ijp-5-5-5.

\section{Introduction}

Due to the Schwarzschild's prediction the term "Black Hole" appeared first use in print due to journalist Ann Ewing in her article "Black Holes in Spaces" as a report on a meeting of the "American Association for the Advancement of Science' [1]. After the use of this term at a lecture from Wheeler in 1967 [2], it was quickly adopted in general use.

It is pointed out all the mass of a Black Hole, $\mathrm{BH}$, were to be compressed within its sphere (see: [3] for detailed description). Since they are that dense there is no mechanism found for providing sufficient outward force to counterbalance the inward pull of gravity, and it seems obvious a BH will collapse into an infinitesimal small size, $i$. e., zero. In nature, however, observations of infinitesimal states in nature are generally questionably more there must be any reason keeping the volume from zero.

A first estimation could be based on the Planck scale [4], [5] to imagine not to correlate more than one Planck mass with each Planck volume, the latter gained from cubic Planck length to show a density

$$
\rho_{P l}=5.1555 \cdot 10^{87} \mathrm{~g} \cdot \mathrm{cm}^{-3}
$$

When related to the solar mass $[6,7]$

$$
M_{\odot}=(1.98855 \pm 0.00025) \cdot 10^{30} \mathrm{~kg}
$$

the value for the Schwarzschild radius $r_{S}$ as the "photon sphere" for a BH of mass $M_{B H}$ can be estimated [8]

$$
r_{S}=\frac{2 G M_{B H}}{c^{2}} \approx \frac{M_{B H}}{M_{\odot}} 2.95 \cdot 10^{3} \mathrm{~m}
$$

The boundary between the interior and exterior of a $\mathrm{BH}$ was surmised the one event horizon. In case BHs lack a surface while acceleration due to gravity increases exponentially situation becomes cumbersome since acceleration is the greatest on a surface of any body. If is assumed a singularity a final value will never reached [9]. If the distribution of matter within the $r_{S}$ had no effect on the outside the actual density of a BH observed from outside might be roughly mass divided volume enclosed within the $r_{s}[8]$

$$
\rho_{B H}=\frac{M_{\odot}}{M_{B H}} 18 \cdot 10^{15} \mathrm{~g} \cdot \mathrm{cm}^{-3}
$$

This relation is exact only for a BH of zero electrical charge and no angular momentum, whereas for more general BH it can differ up to a factor 2 [8].

All those conclusions imply the BHs roughly to demonstrate the same density that will in a constant volume element simply increase with mass. It can be assumed matter sucked into a BH gets crushed into a tiny point at the center interpreted a "singularity" as the only space accumulating everything at a point-matter. Since matter at this point steadily increases the total amount 
must approach infinity. This statement in reverse opens the comparison to the Big Bang, BB as a center of the origin of the universe, where matter comes out leading to the Big Bang Theory, BBT. As a consequence, a BH of infinite density could qualify a "beginning" and therefore lead to another universe associated to expanding. The conclusion is then a reverse of total packing of everything as before. As General Relativity predicts BHs a solution to a set of equations [60], the same theory allows the equations be valid in opposite signs that is a negative $(-)$ to replace the positive $(+)$ instead and reverse.

In this way contradiction appears in cramming positive time intervals $\tau=\Delta t$ to reverse into negative as at the same time reversal in thermodynamic processes means a decrease in entropy, the latter violating the laws of thermodynamics [10]. However, an idea to separate the time coordinate from space exists [11] to therefore cut real space-time behavior as one unit. As it tries to undergo general principle of space-time behavior it became subject to quantum measurements on systems to give experimental evidence of this proposal [12].

In addition to estimations and suggestions to size and further characters of a $\mathrm{BH}$ observed from outside a central question points to the "world" that could be found inside. A principal point of interest is due to a possible change in the character of time and length intervals. However, speculations that way diverge due to the effects revealed in general relativity with the consequence the above variables as well as the speed of light do not behave the same under strong influence of gravitation as in free and influenced space.

Generally, the universe can be seen a flat space [13]. In Einstein's conception space and time are parts of one entity in form of space-time [15], [14] as in Minkowski space (e. $g .,[13,16]$ the linear element of $S$ is defined [15]

$$
d s^{2}=+\left(d X_{0}\right)^{2}-\left(d X_{1}\right)^{2}-\left(d X_{2}\right)^{2}-\left(d X_{3}\right)^{2} .
$$

Then, quantizing gravity implies quantizing space-time leading to a grainy universe, $e$. g., [17].

All those discussions are closely related to a strong coupling between BH theory and the standard BB models, the latter the Friedmann-Lemaitre-Robertson-Walker, FLRW solutions of the gravitational field equations of general relativity [18-25]. These can describe open or closed universes. These FRW universes have a singularity at their beginning, which represents the BB. BHs are required to demonstrate singularities as well, untypical for a mathematical description of nature [26,27]; furthermore, in the case of a closed universe no light can escape, which is just the common definition of it in some accordance to the imagination in Greece mythology.

A couple of decades gave room many scientists to believe our universe is not exclusive besides others. It is assumed a part of a multi-universe and consequently forms one among others in an immense array of separate cosmic universes, and each of them demonstrate its own shining object in the true night sky. A consideration one universe could be linked to another entails a phase highly speculative and missing evident prove or at least a suitable theory. Some researchers have proposed that when a $\mathrm{BH}$ forms, a big bang may occur at the core, which would create a new universe that expands outside of the parent universe [26,28]. In this context the picture of a hyperbolic universe appearing in a negative spatial curvature appears. This type can be thought locally a 3-dimensional analog of an infinitely extended saddle shape, $i$. e., a horn topology as a pseudo-sphere colloquially described as "funnel-shaped" [29].

This trace of thinking represented is obvious, and makes the following study worth to discuss. Since the above cited conclusions expose a $\mathrm{BH}$ a unique gravitational influence the same statement should be possible in consideration of a body consisting of an electromagnetic wave, EM, too. However and in contrast to real matter, light or electromagnetic waves do not present a rest mass, and investigations to a configuration of a $\mathrm{BH}$ other than real matter is missing. Such an intention seems fragile in the view for a mass that could serve the description of the cumbersome properties of light from a classical point of view, since the rest mass of a photon is zero or at least negligible e. g., [30,31,32,33], and theoretical attempts even derive a photon's imaginary mass, e. g., [34]. Moreover, the crux in photon theory $[35,36,37,38,39]$ is the postulate mass-less messenger particles exist from the emission point to the absorption point is not within the four-dimensional space-time continuum; special theory of relativity collapses at the speed of light, because the space-time is shortened to zero to a point so that photon effects properly can not be described by this special theory of relativity.

\section{Theory}

\subsection{Classical Point of View}

Light in the Universe will not become Anti-Light in an Anti-Universe will be kept in mind in this study.

A model is suggested consisting of one EM on a circumference round a theoretical center in a distance $r$ thus interacting with itself via a distance $2 r$ measured from one side to the other.

In the classical view the energy in gravitational interaction between two particles of equal masses $\mathrm{m}$ located in a distance $2 r$ follows from the Newton's law of gravitation [40],

$$
V_{g r}(r)=-G \cdot \frac{m^{2}}{2 r}
$$

with negative energy (attraction) for masses with equal signs or parallel assignment, and positive (detraction) in case of opposite signs or anti-parallel assignment, respectively. In this case the gravitational potential is

$$
\phi(r)=-\frac{G m}{2 r}
$$

It has to be emphasized this $\mathrm{m}$ is not considered a massive mass $\mathrm{M}$ being at rest at the center, and with regard to the denominator $2 \mathrm{r}$ this $\phi(\mathrm{r})$ differs from those incorporated in a system consisting of a relatively small $\mathrm{m}$ moving round an essentially bigger massive mass $M$ located at this center.

Equating expression eq. (6) with [37]

$$
E=m c^{2}
$$




$$
\begin{aligned}
& \left(\text { not }: E=1 / 2 m v^{2} !\right) \text { yields } \\
& \qquad m=\frac{2 c^{2}}{G} \cdot r
\end{aligned}
$$

and the mass is connected to the wave length via the wave-particle dualism $[41,42,43,44]$

$$
m c=\frac{h}{\lambda} \rightarrow m=\frac{h}{c} \cdot \frac{1}{\lambda} .
$$

If a complete wave $\lambda$ interacts with itself the same way compared to a photon eq. (10) interacting with another one located both on opposite sides of the center of a suggested system (Figure 1), the basis condition will define the circumference around it and may consist of a number $n$ of complete waves

$$
2 \pi \cdot r=n \cdot \lambda \rightarrow \lambda=\frac{2 \pi}{n} \cdot r
$$

for a radius $r$. The combination of eqs. (9) and (10) considering eq. (11) draws immediately

$$
r(n)= \pm \sqrt{n \cdot \frac{\hbar G}{2 c^{3}}}
$$

In reverse via eq. (9) the associated mass $m$ of this atom-like system is

$$
m(n)= \pm \sqrt{n \cdot \frac{2 \hbar c}{G}}
$$

which together with eq. (12) appears in the density

$$
\rho(n)=+\frac{1}{n} \frac{6 c^{5}}{h G^{2}}
$$

associated to the complete mass on the surface of that globe giving the numerical

$$
\rho(n)=4.9228378 \cdot 10^{+93} \mathrm{~g} \cdot \mathrm{cm}^{-3}
$$

In spite of its rough approximation the model is consistent with the uncertainty principle

$$
\Delta p_{i} \cdot \Delta r_{i} \geq \frac{\hbar}{2}
$$

[45], which is demonstrated when rewritten in applicant form

$$
p_{i} \cdot r_{i} \equiv+m_{i} c \cdot r_{i}=+\frac{n}{2}
$$

and incorporating the above expressions in eqs. (11) and (12).

The variables in this relation hold for the quality of vectors $\vec{p}$ and $\vec{r}$, respectively both parallel in their directions. Thus, the direction of $r$ determines the sign of $p$ entailing again the sign of $\mathrm{m}$, which finally depend on the sign of the respective components as, e. g., in

$$
(-p)(-r) \equiv(-m) c \cdot(-r)=+\frac{\hbar}{2}
$$

As basis for the next step the condition eq. (9) serves the Schwarzschild radius

$$
r_{S}=\frac{G m}{2 c^{2}}
$$

be determined below. This allows reformation of the associated $\phi(r)$ in eq. (7) into

$$
\phi(a)=-\frac{c^{2}}{a \cdot r_{S}}
$$

as actually measured in units or respective multiples a of $r_{S}$.

It has to be kept in mind that in this classical treatment $\phi$, eqs. (7) and (19), and $r_{S}$, eq. (18), respectively are by the factor 4 lower when compared to the common studies regarding black holes. The reason is that deviation in those studies (a) in the expression for $\phi(r)$ the factor 2 is introduced "by convention" to write

$$
\phi(r)=-2 \frac{G m}{r}
$$

and second, $r$ is the distance between a massive and predetermined defined $M$ and a small test-mass $m$, whereas in the current study $M=\mathrm{m}$ is still a function of $r: m \rightarrow m(r)$; That makes the difference.

\subsection{Incorporating Relativistic Effects}

In the following context the variables are up-indexed "+" and "-" to distinguish between their belongings to the reference frames $S^{+}$and $S^{-}$, respectively.

The expressions for the determination of $r$ and $m$ found for the above model developed under free and undisturbed conditions cannot obey the intention to describe non-inertial movements. When strong gravitational influence appears it comes to the forth the velocity of light propagation or respective speed of a light beam depends on the radius $r$ of the gravitational potential $\phi(r)$ relative to that point of an observer in a frame $S$ at rest and without gravitational influence or at least far from it, $\mathrm{d} \boldsymbol{r}=\mathrm{d} \Omega \equiv 0$, with the speed of light measured $c=c_{0}$. Since the event horizon is insurmountable one part of the EM located on one side of a center can never interact with another on the opposite of a $\mathrm{BH}$, the above "classical" model consequently can not reflect true conditions. Thus, $r$ is now measured from the "surface" of a massive "globe" of mass $M$ to a certain point.

In a first step the variables in the above model must be adjusted following general relativistic properties. The difference principally appears between the expressions for $r$ in direction to the center. Due to

$$
r_{\|}^{\prime} \neq r^{\prime}, \lambda_{\|}^{\prime} \neq 2 \pi r^{\prime}, \Delta r^{\prime}>r_{2}{ }^{\prime}-r_{1}^{\prime}
$$

$r$ used in the former model must be distinctly replaced by the related expressions; it is no longer simply a variable valid in any direction rather it has to be considered individually. It turns out mainly a comparison between the distance $\Delta r$ from $r_{S}$ to the EM as used in the former model to determine $m$ of the photon in eq. (11) becomes a more complicated task since $\Delta r{ }^{\prime}$ and $\lambda^{\prime}$ obey completely different behaviors with regard to the above 
model where simple linear relationship is used. More, a model developed similar to the above is at first certainly appropriate to find an $r_{S}^{+}$outside the event horizon, but in contrast an expression for $r_{S}^{-}$inside an $S^{-}$first must found; it would seam a miracle it is the same amount as $r_{S}^{+}$. The only fact up to now is to indicate the distance $\delta=r_{p H}^{+}-r_{p H}^{-}$between both $S$ to a kind zero-center located outside the insurmountable barriers indicated the associated event horizons. It has to be emphasized up to now $r_{S}^{+}$can be named, but is not still justified by determination. The strategy is suggested thus, to start at the condition allowing an EM one circumference around $r_{S}$ to later take up work on the constitution of $r_{S}$ in reverse.

The radial length contraction for the infinitesimal radial length $\mathrm{d} r$ ' follows from

$$
d r^{\prime}(r)=\sqrt{-g_{11}(r)} \cdot d r \equiv \frac{1}{\sqrt{1-\frac{r_{S}}{r}}} \cdot d r \geq d r
$$

and the time dilation for infinitesimal time interval $\mathrm{d} t$ ' in the same $S$

$$
d t^{\prime}(r)=\sqrt{-g_{00}(r)} \cdot d t \equiv \frac{1}{\sqrt{1-\frac{r_{S}}{r}}} \cdot d t<d t
$$

(Einstein, 1916). From eq. (22) any distance to the event horizon is

$$
\begin{aligned}
& \Delta r^{\prime}(r)=\int_{r_{1}}^{r_{2}} d r_{\|}^{\prime}(r)>r_{2}-r_{1} \\
& \rightarrow \\
& \Delta r^{\prime}(r)=r_{S} \sqrt{\frac{r}{r_{S}}}+\frac{r_{S}}{2} \ln \left[2 \frac{r}{r_{S}}-1+2 \sqrt{\frac{r}{r_{S}}\left(\frac{r}{r_{S}}-1\right)}\right]
\end{aligned}
$$

the proper-radial length.

For a time interval $\Delta t^{\circ}(r)$ on the sphere (perpendicular to $r$ ) in a frame $S$ ' relative to a time interval $\Delta t$ in a frame $S$ outside follows with regard to eq. (23)

$$
\Delta t^{\prime}(r)=\sqrt{1-\frac{r_{S}}{r}} \Delta t \leq \Delta t
$$

Consequently, an EM oscillation in a certain propertime period $T^{\prime}$ takes longer, works reciprocally to its frequency

$$
v^{\prime}(r)=\frac{1}{T(r)}=\sqrt{1-\frac{r_{S}}{r}} \cdot \frac{1}{T_{0}}=\sqrt{1-\frac{r_{S}}{r}} \cdot v_{0}<v_{0}
$$

to yield the wave length

$$
\lambda^{\prime}(r)=\frac{1}{\sqrt{1-\frac{r_{S}}{r}}} \cdot \lambda_{0}>\lambda_{0}
$$

and associated mass

$$
m^{\prime}(r)=\frac{1}{\sqrt{1-\frac{r_{s}}{r}}} \cdot m_{0}>m_{0}
$$

It can be realized eq. (27) directly refers to a distance $s$ in the curved space with an infinitesimal length element $\mathrm{d} s$ (r) orthogonal to $\mathrm{d} r^{\prime}(r), i$. e., $d s^{\prime}(r) \perp d r r^{\prime}(r)$. In replacing $r$ by $r^{\prime}{ }_{\perp}(r)$ to emphasize its character valid perpendicular to $r$ and to avoid confusion with $\Delta r^{\prime}{ }_{/ /}(r)$ makes eq. (27) turn into the to $s$ associated radius (Figure 1)

$$
r_{\perp}^{\prime}(r)=\frac{1}{\sqrt{1-\frac{r_{S}}{r}}} \cdot r
$$

A combination of this expression together with eq. (24) should lead to a determination of the photon radius or photon sphere $r_{p H}$, respectively. It has to be emphasized, however, the variable $r$ in eq. (29) refers to the distance to the center whereas $r$ in the expression for $\Delta r^{\prime} /(r)$ of eq. (24) refers to the distance to the sphere of event horizon at $r_{S}$. Therefore, the variable $r$ in the latter is based on $r_{S}$ and must be replaced $r=r+r_{S}$ to allow comparison with eq. (24) then renamed

$$
\begin{aligned}
\Delta r_{\|}^{\prime}(r) & =\Delta r^{\prime} \|\left(r+r_{S}\right)=r_{\|}^{\prime}(r)^{+} \\
r^{\prime}{ }_{\|}(r)^{+} & =r_{s} \sqrt{\frac{r+r_{s}}{r_{s}}}+\frac{r_{s}}{2} \ln \left[2 \frac{r+r_{s}}{r_{s}}-1+2 \sqrt{\frac{r+r_{s}}{r_{s}}\left(\frac{r+r_{s}}{r_{s}}-1\right)}\right]
\end{aligned}
$$

or depending on the dimensionless variable $a=r / r_{S}$ as measured in units of $r_{S}$

$$
\begin{aligned}
& r_{\|}^{\prime}(a)^{+}=r_{S}\left(\sqrt{a+1}+\frac{1}{2} \ln [2(a+1)-1+2 \sqrt{a(a+1)}]\right) \\
& \longrightarrow \quad r^{\prime}{ }_{\|}(a)^{+}=r_{S}\left(\sqrt{a+1}+\frac{1}{2} \ln [2 a+1+2 \sqrt{a(a+1)}]\right)
\end{aligned}
$$

respectively. The intersection of $r_{\|}^{\prime}(r)$ with $r_{\perp}^{\prime}(r)$ is determined then (Figure 1)

$$
\frac{a^{*}}{\sqrt{1-1 / a^{*}}}=\left(\sqrt{a^{*}+1}+\frac{1}{2} \ln \left[2 a^{*}+1+2 \sqrt{a^{*}\left(a^{*}+1\right)}\right]\right)
$$

and the factor $a^{*}(r) \approx 1.4761581$ found via iteration corresponds to the photon sphere at

$$
r_{p H} \approx 1.4761581 \cdot r_{S} .
$$

Due to the distinct behavior in radius dependence of the eqs. (28), (29) the following prove is exclusively valid for the fixed factor $a^{*}$ to produce their point of intersection in accordance to eq. (32). Then, the eqs. (10) and (11) with respect to the transformations by eq. (27) and (28), respectively create the relationship

$$
m^{\prime}(r)=\frac{h}{c \lambda}, \equiv \frac{h}{2 \pi c r}, \equiv \frac{\hbar}{c r},
$$

and after joining again with eq. (9) with renamed variables then displays perfect agreement with the results found before. 
This demonstration sustains the result found above with respect to the variables and now under their respective relativistic transformation. In spite of that from the beginning of this context on it was not alone the intention to find an $r_{p H}$ but even this was the basis. As already mentioned the current model properly deals with the "inside conditions" within the "inside shell" of the $\mathrm{BH}$, and an expression for $r_{S}$ has only been conjectured in eq. (19). Since there is no massive $M$ but rather $r$ and $m$ resulting as variables from the basic condition alone there could be an anticipated relation let up from $r$ in eqs. (12) to $r_{S}$. This can be proven when dividing eq. (12) by the expression for $r_{S}$ in eq. (18) and then replacing $m$ from eq. (13). It shows an

$$
r^{\prime}(r)= \pm \sqrt{\frac{\hbar G}{2 c^{3}}}= \pm r_{P H}
$$

which is in accordance to the model presented above and points to a real

$$
\left(r_{P H}\right)^{ \pm}= \pm 11.428463 \cdot 10^{-36} \mathrm{~m}
$$

outside and respective inside a $\mathrm{BH}$ as surmised. Since $a^{*}$ as always positive justifies condition eq. (32) an expression for $r_{S}$ as the central point displays immediately

$$
\begin{aligned}
\left(r_{S}\right)^{+} & =+\frac{r_{P H}}{a^{\star}} \equiv+\frac{1}{a^{\star}} \sqrt{\frac{\hbar G}{2 c^{3}}} \approx+\frac{1}{1.4761581} \sqrt{\frac{\hbar G}{2 c^{3}}} \\
\rightarrow \quad\left(r_{S}\right)^{+} & \approx+7.78 \cdot 10^{-36} \mathrm{~m}_{(36)}
\end{aligned}
$$

This is a mass-independent solution and obviously valid in both $S^{\prime}$, outside as inside a $\mathrm{BH}$ as well being the result from exclusively an EM alone originated from itself and without any preliminarily given massive $M$ dominating the system (Figure 2). The values are real, theoretically measurable, and exclude be imaginary. Since both signs are possible and appear immediately equal valued it can be surmised the two frames $S^{\prime}$ exactly opposite to each other in behavior.

Generally, the statement could not be pointed out if the expressions eqs. (35) or (36), respectively were determined by any variable depending eventually then on any external influences; that was the reason for the extended relativistic study to then justify those expression valid in any frame.

\subsection{The Space Inside}

In further continuation of this work the task is to look for some symmetry between the frames outside and inside a $\mathrm{BH}$ to consider the related transformation equations inside an $S^{-}$. A way to derive comparison between positive and negative states can be seen similarly to the interpretation of the Dirac's theory [46], [47] since it allows or even demands for negative states. On the basis of the above experiences of the current investigation an appropriate formalism must be achieved to provide a way to translate the fields and forces from one frame to another.

Obviously, space-time can be displayed for example the fourth vector

$$
S^{+}=\left[\begin{array}{l}
+i X_{0} \\
+X_{1} \\
+X_{2} \\
+X_{3}
\end{array}\right] \equiv\left[\begin{array}{l}
+i c t \\
+X \\
+Y \\
+Z
\end{array}\right]
$$

and completely reversed in accordance to $C P T$-operation

$$
S^{-}=\left[\begin{array}{l}
i X_{0} \\
-X_{1} \\
-X_{2} \\
-X_{3}
\end{array}\right] \equiv\left[\begin{array}{l}
i c t \\
-X \\
-Y \\
-Z
\end{array}\right]
$$

as well, but the both frames require two distinct types of transformation equations.

Of course, every task again only holds in case of an attracting gravitational force leading in a first view to the condition both $m$ in eq. (6) are the same sign. If their signs are different their distance $2 r$ must be negative to then compensate this fact in making the energy keep positive sign. Otherwise, this would contradict to an existence of the "negative" $r_{p H}^{-}$, which truly occurs as indeed given in eq. (35). If however the variables $\mathrm{r}$ and $\mathrm{m}$ were considered instead of a distance $(2 r)$ together with $\left(m^{2}\right)$ in eq. (6) this will not ban the possibility of negative signs, since it would affect both of them at the same time. This is justified by the Heisenberg's uncertainty eq. (17). Consequently, all variables in $S^{-}$can display also in negative sign and keeping the sign for the gravitation potential eq. (6) negative at any rate, thus.

The argument voting for reciprocal behavior in a transformation $S^{+} \leftrightarrow S^{-}$arises from the reason any value $r$ can take up in distance within its respective $S^{-}$is strictly limited the same manner as in $S^{+}$. Taking into fact those variables are properly freely selectable it requires in spite of that respect to their restriction due to the true existence of $\left|r_{p H}\right|$ as entailed from the fundamental condition eq. (32). The latter is a condition in itself and in addition justified with regard to the constant $a^{*}$. Obviously, this condition will be always obeyed if a variable changes in value or respective amount as from $r$ into a ratio $1 / r$.

As now established those two statements can be summarized as the configuration space to space-time curvature in both frames $S^{\prime}$ demonstrate opposite behavior to each other in direction and completely reverse in behavior. The mathematical design for the transforming of the space-time coordinates in reversed form arises then

$$
\left(X_{i}^{+}\right)^{+1} \rightarrow\left(X_{i}^{-}\right)^{-1}
$$

The variables are restricted in their absolute values

$$
r_{S}^{+} \leq\left|r^{+}\right| \leq \infty \text { and } 0 \leq\left|r^{-}\right| \leq r_{S}^{-}
$$

where their sequences display the intervals from either the event horizon in the $S^{+}$outside a BH to infinity or from zero to the event horizon inside. The above scheme is taken for use in a transformation regarding the peculiar properties of the frames to arrive at the physical properties in the respective reference frames. 
Due to the rules eqs. (40) and (41) compared to those for $S^{+}$the essential transformation equations for $S^{-}$can be explicitly given for $r$ and the units

$$
b \cdot r_{S}^{-}=r^{-} \text {with } 0 \leq b \leq 1,
$$

respectively. Those read with respect to length contraction

$$
\begin{aligned}
& d r^{\prime}\left(r^{-}\right)=-\frac{1}{\sqrt{1-\frac{1 / r_{S}^{-}}{1 / r^{-}}}} \cdot d r=-\frac{1}{\sqrt{1-\frac{r^{-}}{r_{S}^{-}}}} \cdot d r \leq \cdot d r^{-} \\
& \rightarrow d r^{\prime}\left(r^{-}\right)=-\frac{1}{\sqrt{1-b}} \cdot d r,
\end{aligned}
$$

time dilation

$$
d r^{\prime}\left(r^{-}\right)=-\sqrt{1-b} \cdot d r,
$$

and for the path $s$ perpendicular to $r$

$$
r_{\perp}^{\prime}\left(r^{-}\right)=-\frac{b}{\sqrt{1-b}} \cdot d r \leq r_{\perp}\left(r^{-}\right) .
$$

Analogously to eq. (24) the distance from the event horizon displays

$$
\begin{aligned}
& \Delta r_{\|}^{\prime}(r)=\int_{\eta^{-}}^{r_{2}^{-}} d r^{\prime} \|\left(r^{1}\right)>r_{2}^{-}-r_{1}^{-} \\
& \rightarrow \\
& \Delta r_{\|}^{\prime}(r)^{-}=-2 r_{S} \cdot \sqrt{1-\frac{r^{-}}{r_{S}^{-}}}
\end{aligned}
$$

or incorporating $b$ in the respective form

$$
\Delta r^{\prime} /\left(r^{-}\right)^{-}=-2 r_{S} \cdot \sqrt{1-b}
$$

The variable $r$ in eq. (45) refers directly to the distance from the sphere at $r_{S}^{-}$to the origin, because in contrast to the conditions in the $S^{+}$the event horizon in the $S^{-}$is located outside the $r_{p H}$ (Figure 2). Consequently, there is no barrier to over-jump and a connection between the radii $r_{S}^{-}$and $r_{p H}$ can directly be established equating eqs. (44) and (45) to reveal another constant together with the event horizon from the eqs. (35), (36) inside a $\mathrm{BH}$ to further give evidence for the reciprocal behavior between both $r_{S}$,

$$
\begin{aligned}
& \frac{b^{*}}{\sqrt{1-b^{*}}} \cdot r_{S}^{-}=2 \sqrt{1-b^{*}} \cdot r_{S}^{-} \\
& \rightarrow b^{*}=\frac{2}{3} \approx \frac{1}{a^{*}}
\end{aligned}
$$

and the associated Schwarzschild radius

$$
\begin{aligned}
\left(r_{S}\right)^{-} & =-\frac{r_{P H}}{b^{\star}} \equiv-\frac{1}{b^{*}} \sqrt{\frac{\hbar G}{2 c^{3}}} \approx-\frac{3}{2} \sqrt{\frac{\hbar G}{2 c^{3}}} \\
\rightarrow\left(r_{S}\right)^{-} & =-7.6189754 \cdot 10^{-36} \mathrm{~m}
\end{aligned}
$$

\subsection{Comparison of the Physical Quantities in the Frames}

The investigation up to now considers two frames separatly instead of incorporating both frames into a single one, which is properly the aim of the current investigation as to look at the properties of the $\mathrm{BH}$ ocurring inside. It has been pointed out $r_{S}^{+}$and $r_{S}^{-}$are reciprocal to eachother due to their origin from $r_{P H}$ via the above transformation (Figure 3). As both $r_{p H}$ are inmidiately touching themselves the same is true for both of the $r_{S}$ (Figure 3), and the above prognosticated "miracle" has become true. It has to be emphazised, however that statement has to be carefully considered in the view the two radii being reciprocal to each other, and the scaling is not the same. It is, therefore $r_{S}^{+}=1 / r_{S}^{-}$rather than an absolute comparison between both of the values. A true comparison of the frames is not purely possible yet since in both frames still the same and antropogenic units for the variables are used, which bans evaluation of their physical properties on a unified basis..

Obviously the main focus is to look towards an appropriate scaling with the property to evaluate those frames $S^{+}$and $S^{-}$both rating free so not to violate the principle of equal rating. Those rating-free options requires a system based on only the properties of free space and free of antropogenic arbitrariness to allow a unified statement. Such a system has already been developed in the Planck's scale [4] and based on natural units. A non-anthropogenic solution will then provide a frame $S^{0}$ as being neutral as not to favor any other frame, $i$. e., the one inside or the one outside a $\mathrm{BH}$ either, when compared in their characteristics.

The current investigation follows that proposal to take the Schwarzschild radius $r_{S}$ as the uniform reference for the units to explain the world inside a BH in a vis-a-vis study. As a consequence it will be advantagous to define it a unity pointing to the relation

$$
r_{S}=1 \equiv\left|a^{*} \cdot r_{S}^{+}\right| \equiv\left|b^{*} \cdot r_{S}^{-}\right| \equiv \sqrt{\frac{\hbar G}{2 c^{3}}}
$$

which in revers points again to the basis concept used to even develop the entire study. The unit for the mass is already given in eq. (13) and that for a time interval derived from eq. (48) incorporating the speed of light

$$
\Delta t= \pm \sqrt{\frac{\hbar G}{2 c^{5}}}
$$

Of course, both options in sign are possible.

However, a look towards the physical properties opens a more complex situation, and needs further investigation for an indepth understanding of the world inside a BH. An example is demonstrated on the pair of the Dirac's hamiltonians resulting from the Poisson's equation,

$$
\mathcal{H}= \pm\left(-i \hbar c \vec{\alpha} \nabla+\beta m_{0} c^{2}\right) \pm V(r)
$$

with the lowest energy state of an $\mathrm{H}$-like atom, e. g., [48]

Quod erat demonstrandum. 


$$
E=\mp\left(m_{0} c^{2}\right) \cdot \sqrt{1-(Z \cdot \vec{\alpha})^{2}}
$$

Obviously, the kinetic part in the pair of Hamiltonians representing the free particle can be symmetrically transformed into each another with respect to their sign, whereas the potential $V(r)$ strictly emphasizes their different behavior. That demands to carefully distinguish those facts when the signs of the mass or the electric charge are simply tried to interchange. The Hamiltonians are derived from an EM as well as the basis principle in this theory and must be therefore inside the frame $S^{-}$of a $\mathrm{BH}$ interpreted the same manner as outside. The negative sign at the potential $V$ in eq. (51) is always true due to the electrostatic attraction between nucleus and electron; a reverse in sign of their charges does not affect this. As a consequence, a stable character of an atom in a world of variables of negative sign can be strictly concluded.

\section{Results}

Due to the object of the current study the main result presents in the transformation equations those allow to compare the physical characteristics in a frame $S^{-}$inside a BH with those outside, $i$. e., the "real" world. It can be stated outboth $r_{S}$ as well as both $r_{p H}$ inside and outside a $\mathrm{BH}$ touch eachother (Figure 3). In addition the theory allows the evaluation to size and mass of a $\mathrm{BH}$ togther with the associated density as well as the units for the length and time interval are obtained. The numerical values obtained respective inside and outside a $\mathrm{BH}$ can appear in positive and negative signs and show the Schwarzschild radius

$$
\left(r_{S}\right)^{-}=-7.6189754 \cdot 10^{-36} \mathrm{~m} \text { and }\left(r_{S}\right)^{+}=+7.78 \cdot 10^{-36} \mathrm{~m} \text {, }
$$

photon radius or sphere, respectively

$$
\left(r_{P H}\right)^{ \pm} \approx \pm 11.428463 \cdot 10^{-36} \mathrm{~m}
$$

and the smallest time interval

$$
\Delta t= \pm 3.8121249 \cdot 10^{-44} \mathrm{~s}
$$

Finally, mass and density of a $\mathrm{BH}$ inside and outside are

$$
\begin{gathered}
m= \pm 30.77993 \cdot 10^{-9} \mathrm{~kg}, \\
\rho=+4.9228378 \cdot 10^{+93} \mathrm{~g} \cdot \mathrm{cm}^{-3},
\end{gathered}
$$

respectively, where obviously the density is exclusively positive.

In conclusion, the frames inside a $\mathrm{BH}$ and outside are related to eachother incorporating via the relativistic transformation equations refering to the space-time coordinates

$$
\left(X_{i}^{+}\right)^{+1} \rightarrow\left(X_{i}^{-}\right)^{-1}
$$

pointing to a complete reversal of the physical conditions when comparing the frames in- and outside a $\mathrm{BH}$.
Due to the conditions entailed alone from the transformations for length and time itself any length divided by time keeps the direction in velocity, and will not affect the propagation of a light beam that way, either. However, a reversal in sign of the mass turns matter into anti-matter, the latter will be attracted in direction to the $r_{S}^{-}$appearing as anti-matter as well making the anti-particle grow in acceleration. The consequence is a particle of positive mass entering a $\mathrm{BH}$ appears inside in character of an anti-particle in the center to propagate then in direction of the sphere presented by the inside $r_{S}^{-}$ (Figure 3). This emphasizes the imagination of an acceleration growing in direction to the sphere enveloping its inside.

Since from an observer in an $S^{0}$, far away and outside the $\mathrm{BH}$ the center of a $\mathrm{BH}$ is evaluated 0 or respective zero this becomes infinite under the length transformation. Thus, a BH demonstrates an anti-universe inside of the same conditions compared to the universe, and here the effect of the $\mathrm{BH}$ to swallow everything becomes true as well (here it would be an anti-BH). As a conclusion, both $r_{S}$ demonstrate the same character inside and outside the $\mathrm{BH}$. Essentially, a BH can be imagined a tiny globe to envelope an almost empty space, which consists of its own universe getting more and more (anti-)mass as external mass is sucked into from the outside; an observer inside the frame $S^{-}$would therefore consider the kinetic behavior as originating mass.

\section{Discussion}

On a first view, it seems improbable to achieve an approach to answer to the question for the inside of a $\mathrm{BH}$ since it is stated insurmountable. It is fact under general circumstances it is, but the way suggested here truly opens the field for a discussion and statistical interpretation of the physical properties on an expected world inside. As a consequence it could be highlighted in this study an observer in the exerted frame $S^{+}$outside a $\mathrm{BH}$ will therefore consider a frame $r_{S}$ making the outside the inner just flipping everything. The transformation equations leave this fact unambiguous. In spatial coordinates the two frames can be imagined a three-dimensional "cutting surface" of a four-dimensional globe. The space inside both frames is transparent, an observer at one side theoretically able to view the other one on the opposite.

From the point of view for an outside observer the actual $r_{S}$ and $\rho$ of a $\mathrm{BH}$ can be estimated due to eqs. (3) and (4), respectively. That makes sense, since the distribution of matter within the $r_{S}$ as the membrane around has no effect on the outside. With respect to the numerical values given from the Planck's scale and those estimated from the eqs. (3), (4)

$$
\begin{gathered}
l_{\text {Planck }}=16.16199(97) \cdot 10^{-36} \mathrm{~m}, \\
r_{S \text { Wald }}=4.6436872 \cdot 10^{-36} \mathrm{~m},
\end{gathered}
$$




$$
\begin{aligned}
& \rho_{\text {Planck }}=5.15500 \cdot 10^{+87} \mathrm{g.cm}{ }^{-3}, \\
& \rho_{\text {Wald }}=75.125799 \cdot 10^{+90} \mathrm{~g} . \mathrm{cm}^{-3},
\end{aligned}
$$

show the associated values achieved in the current study display approximately agreement in the $r_{S}$ whereas that for the density gained from the above estimation formulas is a factor 100 lower. An explanation for the disagreement in the latter could be settled in the mass, which in the actual study is derived alone from the EM.

The present investigation is based on photon theory of special relativity bearing the postulate mass-less messenger particles moving from the emission point to the absorption point, and this is properly not within the four-dimensional space-time continuum. It is true, space-time is shortened to zero to a point so that photon effects can not be described by this special theory of relativity, which collapses at the speed of light. However, it has already pointed out light in the vacuum propagates with a constant speed $c$, but this speed is determined by two alternating speeds altogether and can be interpreted oscillating between them interchanging sub- and superluminal movements $[49,50]$. In the view of the photon itself the Proca's theory [34] has been derived also an oscillating photon mass. Those results point to the fact time does not stand still at the speed of light but rather oscillates, too. In a way the time direction is bent more and more inwards as getting closer to the $\mathrm{BH}$ and becomes negative in crossing the event horizon. In a way, the time direction is bent more and more inward as you get closer to the black hole.

A consequence of the free rating of both of the frames entails some more interesting matter. Though, it is true no matter can escape from both sides of the $r_{S}$ if it approaches close enough, the main question still remains where all that matter will be collected after sucked into the $\mathrm{BH}$. In considering the case a particle can really pass the $r_{S}$ barrier it will appear in the center; it is excluded this particle keeps staying close to the $r_{S}$ because under those circumstances it would be again captured. For an observer inside the frame $S^{-}$this effect will display as a growth in the here negative mass (Figure 3).

Another point of view in that context is due to thermodynamic considerations. It has been argued under this aspect a decrease in entropy must be expected just entailing the enthalpy to become negative $[10,13,51,52,53]$. Apart from this statement found on statistical considerations an observer in a frame $S^{+}$or $S^{0}$, respectively outside a BH will assure time reversal as time inside the frame $S^{-}$ runs anti-clockwise. Consequently, a time reversal stated in the current paper does not disregard that aspect or statistical interpretations, either. The symmetry found between the two frames emphasized here is indeed in agreement with an asymmetry found on past-future considerations [54]. It has been shown indeed even a kind decoupling between space and time might be possible [11].

An example for the mirroring in space-time inside a $\mathrm{BH}$ can be the pair of Dirac's equations. Since they provide both characters in sign any "trouble" in their treatment becomes exactly the same inside as outside a $\mathrm{BH}$, but allows determination in quantum mechanical studies the same way.

Besides speculations and even philosophical aspect contributing discussions it is obvious thinking that way opens again further ranging questions. Although, evidence for a real existence of BHs is not proven and there are arguments against that [55] it has been claiming instead there are "apparent horizons" sucking matter and energy only temporarily and before they release them again [56]. With respect to the concentration of the mass of a $\mathrm{BH}$ to a point zero is valued a contradiction in the existence of singularities in nature $[53,57,58]$, though especially quantum field theory deals with that fact. Further, unitarity is the part of quantum theory that strongly disapproves of event horizons being a point of no return.

The results in the presented paper agree with the aspect space and time change places at the event horizon the way they reverse leading to fundamental consequences. A central question appears in the characterization of acceleration comparing both sides of the event horizon together with the question where the mass captured into a $\mathrm{BH}$ remains. Since none of the both frames is exposed against the other it would be possible to predict growing acceleration as a particle approaches the event horizon. On the other side a backward running time will turn the sign in acceleration into negative meaning deceleration with the consequence a mass particle entering a $\mathrm{BH}$ would appear then in the center of it to become slower in direction to the event horizon. The latter argument would then open the imagination of a white hole [59] just originating mass from obviously nothing. In reverse, here the energy of the particles must be enormous, though negative, but a strong argument then holds in the reverse in space speaking against the fact of deceleration but argues for a reverse in the acceleration direction. In addition, the transformation of the fourth vector in spacetime points to the fact of increasing expansion of space with approaching the center inside. As a conclusion, both observations would be possible as a white hole could be the place of bearing matter, in that case a respective antimatter, of enormous negative energy slowing down in direction to the event horizon or the matter, which has entered the $\mathrm{BH}$ will appear at the event horizon inside with high speed due to its energy. Of course, this energy must be negative since the mass turns in sign. From the perspective of an observer inside a $\mathrm{BH}$ all matter then races from all directions. Even the source universe may become a remote eternity away. Those circumstances could certainly incorporate the possibility for an explosion of a $\mathrm{BH}, e ., g .$, [52]. Generally, a $\mathrm{BH}$ can essentially imagined an empty globe representing an anti-universe striving the way from a cumulative mass distribution into a flat space, meaning expansion, the same as the "real" universe in nature.

The central point, however, remains the two frames can be imagined two globes of physical characters oppositely in sign and character, but should allow free rating evaluation. An expansion in direction to the center is therefore a mathematical consequence and therefore bans a then possibly appearing collapse of this anti-universe inside, which would be the analogy to a discussed collapse of the $\mathrm{BH}$ in general. 
From this point of view a difference between the BB singularity of the FRW models and that of a $\mathrm{BH}$ becomes obvious as the first lies in the past, whereas the second in the future. A white hole then is considered a time-reversed version of a $\mathrm{BH}$, a statement, which incorporates the investigations in the current paper.

\section{Conclusion}

A new model for a $\mathrm{BH}$ is suggested to allow to study the physical properties inside. Here, the basis consists of an EM wave interacting with itself via gravitational forces. First, classical treatment is given to further expand the theory to general relativistic considerations. Due to the model character there is no predominantly given massive mass $M$ in contrast to earlier studies.

In the theory part of the current study a set of relativistic transformation equations were derived, which provide the possibility required to evaluate the conditions inside a $\mathrm{BH}$ as well as a later comparison between the two frames appearing inside and outside. The results are remarkable due to the fact two respective individual values for the Schwarzschild radii and the photon spheres, respectively were found. It could be shown those touch each another in spite of their belongings to two distinct frames.

A consequence of the model character was to open a way to imagine the character inside as to evaluate that space as an inversed universe where the variables hold following $C P T$-operation. This led to demonstrate the inside of a $\mathrm{BH}$ mirrored in the variables of space-time. It was found mass and radius inside the $\mathrm{BH}$ become negative as well as physical processes run backward in time. Another aspect even pointed to the appearance of anti-matter, which results from the negative sign of the mass.

The present work theory was appropriate to provide a calculus for determination of the radius, mass, and density of a $\mathrm{BH}$, which enables a scientist to exactly determine the structural behavior inside a $\mathrm{BH}$ and allows free rating evaluation of this world inside.

A final insight to the possible path in the origin of a universe could be given to establish a fundament for further discussion in that matter.

\section{References}

[1] Quinion, M. Black Hole. World Wide Words. (2008).

[2] Misner, C.; Thorne, K. S.; Wheeler, J. Gravitation. W. H. Freeman and Company. (1973).

[3] Chandrasekhar, S. Mathematical Theory of Black Holes. Oxford University Press. (1999).

[4] Planck, M. Ueber irreversible Strahlungsvorgaenge. Sitz.ber. Koenigl. Preuss. Akad. Wiss. Berlin 5 (1899). 440-480.

[5] Tomilin, K. A. Natural Systems of Units: To the Centenary Anniversary of the Planck System. Proceedings Of The XXII Workshop On High Energy Physics And Field Theory (1999). 287-296.

[6] Harwit, M. Astronomical concepts. Astronomy and astrophysics library (3rd ed.). Springer (1998). pp. 72-75.

[7] Mohr, P. J.; Taylor, B. N.; Nevell, D. B. CODATA Recommended Values of the Fundamental Physical Constants: 2006. Rev. Mod. Phys. 80 (2008). 633-730.
[8] Wald, R. M. General Relativity. University of Chicago Press. (1984).

[9] Schwarzschild, K. Ueber das Gravitationsfeld eines Massenpunktes nach der Einsteinschen Theorie. Sitzungsberichte der Koeniglich Preussischen Akademie der Wissenschaften zu Berlin 1 (1916). 189-196.

[10] Hawking, S. W. Black Holes and Thermodynamics. Phys. Rev. D 13 (1976). 191-187.

[11] Goedel, K. An Example of a New Type of Cosmological Solutions of Einstein's Field Equations of Gravitation. Rev. Mod. Phys. 21 (1949). 447-450.

[12] Foroozani, N.; Naghiloo, M.; Tan, D.; Murch, K. Correlations of the Time Dependent Signal and the State of a Continuously Monitored Quantum System. Phys. Rev. Lett. 116. (2016). 110401.

[13] Wheeler, J. A. A Journey into Gravity and Spacetime. Scientific American Library, W. H. Freeman \& Co, New York (distr.) New York. (1990).

[14] Hilbert, D. Die Grundlagen der Physik. Koenigliche Gesellschaft der Wissenschaften zu Goettingen, Mathematisch-Physikalische Klasse, Nachrichten. (1915). 395-407.

[15] Einstein, A. Die Grundlage der allgemeinen Relativitaetstheorie. Ann. Phys. 49. (1916). 769-822.

[16] Faber, R. L. Differential Geometry and Relativity Theory: An Introduction. Marcel Dekker, Inc., New York, N. Y. (1983).

[17] Cao, Z.; Cao, H. G. Unified Field Theory and the Hierarchical Universe. Int. J. Phys. 1. (2013). 162-170.

[18] Friedmann, A. Ueber die Kruemmung des Raumes. Z. Phys. A 10 (1922) 377-384

[19] Friedmann, A. Uebr die Moeglichkeit einer Welt mit konstanter negativer Kruemmung des Raumes. Z. Phys. A 21. (1924). 326-332

[20] Lemaître, G. Un universe homogène de masse constante et de rayon croissant rendant compte de la vitesse radiale des nébuleuses extra-galactiques. Annales de la Société Scientifique de Bruxelles A 47. (1927). 49-56.

[21] Lemaître, G. l'Universe en expansion. Annales de la Société Scientifique de Bruxelles A 53. (1933). 51-85.

[22] Robertson, H. P. Kinematics and world structure. Astrophysical J. 82. (1935). 284-301

[23] Robertson, H. P. Kinematics and world structure II. Astrophysical J. 83. (1936a). 187-201.

[24] Robertson, H. P. Kinematics and world structure III. Astrophysical J. 83. (1936b). 257-271

[25] Walker, A. G. On Milne's theory of world-structure. Proc. Lond. Math. Soc. 2 42. (1937). 90-127.

[26] Poplawski, N. J. Radial motion into an Einstein-Rosen bridge. Phys. Lett. B 687. (2010). 110-113.

[27] Poplawski N. J. Nonsingular, big-bounce cosmology from spinortorsion coupling. Phys. Rev. D 85. (2012). 107502.

[28] Fahri, E.; Guth, A. H. An Obstacle to Creating a Universe in the Laboratory. Phys. Lett. B 183. (1987). 149-155.

[29] Aurich, R.; Lustig, S.; Steiner, F.; Then, H. Hyperbolic Universes with a Horned Topology and the CMB Anisotropy. Classical Quantum Gravity 21. (2004). 4901-4926.

[30] Adelberger, E., Dvali, G., Gruzinov, A. Photon-Mass Bound Destroyed by Vortices. Phys. Rev. Lett. 98. (2007). 2019-1028.

[31] Amsler, C. et. al. (Particle Data Group). Phys. Lett. B 667. (2008). 1-6.

[32] Chibisov, G. V. Astrophysical upper limits on the photon rest mass. Soviet Physics Uspekhi 19. (1976). 624-626.

[33] Pound, R.; Rebka, G. Apparent Weight of Photons. Phys. Rev. Lett 4. (1960). 337-341.

[34] Proca, A. Sur la théorie ondulatoire des électrons positifs et négatifs. J. Phys. Radium. 7. (1936).347-353

[35] Einstein, A. Ueber einen die Erzeugung und Verwendung des Lichtes betreffenden heuristischen Gesichtspunkt. Annalen der Physik 17. (1905a). 132-148.

[36] Einstein, A. Zur Elektrodynamik bewegter Koerper. Annalen der Physik 17. (1905b). 891-921.

[37] Einstein, A. Ist die Traegheit eines Koerpers von seinem Energiegehalt abhaengig? Annalen der Physik 18 (1905c). 639641

[38] Einstein, A. Ueber das Relativitaetsprinzip und die aus demselben gezogenen Folgerungen. Jahrbuch der Radioaktivitaet und Elektronik IV. (1908). 411-462. 
[39] Einstein, A. Ueber die Entwicklung unserer Anschauungen ueber das Wesen und die Konstitution der Strahlung, Phys. Z. 10 .(1909). $817-825$.

[40] Newton, I. Principia Mathematica Philosophie Naturalis (1686) (Reprinted by University of California Press, Berkeley, California (1934).

[41] De Broglie, L. V. Rayonnement noir et quanta de lumière. Journal de Physique et le Radium 3. (1922a). 422-428.

[42] De Broglie, L. V. Sur les interférence et la théorie de quanta de lumière. Compt. Ren. 175. (1922b). 811-813.

[43] De Broglie, L. V. Recheres sur la theorie des quanta. Ph. D. Thesis. Faculté des Sciences, Université de la Sorbonne, Paris (1923a).

[44] De Broglie, L. V. Ondes et Quanta. Compt. Ren. 177. (1923b) 507-509.

[45] Heisenberg, W, K. Über quantentheoretische Umdeutung kinematischer und mechanischer Beziehungen Z. Phys.83. (1925). 879-893.

[46] Dirac, P. A. M. The Quantum Theory of the Electron. Proc. Roy Soc. A 117. (1928a). 610-624.

[47] Dirac, P. A. M. The Quantum Theory of the Electron. Part II. Proc. Roy. Soc. London A 118. (1928b). 351-3611.

[48] Schwabl, F. Quantenmechanik fuer Fortgeschrittene (QM II), 5-th ed. Springer, Berlin \& Heidelberg, 2008.

[49] Gerlitz, T. G. M. Superluminality and Finite Potential LightBarrier Crossing. Int. J. of Research in Pure and Applied Physics 5. (2015a). 19-24.
[50] Gerlitz, T. G. M. The Mysterious Finestucture Constant $\alpha$ in Quantum Mechanics. Advanced Engineering and Applied Sciences. An International Journal. 5. (2015b). 79-82.

[51] Bardeen, J. M. ; Carter, B.; Hawking, S. W. The four laws of black hole mechanics. Comm. Math. Phys. 31. (1973). 161-170.

[52] Hawking, S. W. Black hole explosions? Nature 248. (1974). 30-31.

[53] Hawking, S. W.; Penrose, R. The Singularities of Gravitational Collapse and Cosmology. Proc. Royal Soc. A 314. (1970). 529-548.

[54] Finkelstein, D. Past-Future Asymmetry of the Gravitational Field of a Point Particle. Phys. Rev. 110. (1958). 965-967.

[55] Israel, W. Event Horizons in Static Vacuum Space-Times. Phys. Rev. 164. (1967). 1776-1781.

[56] Israel, W. Dark stars: the evolution of an idea. 300 Years of Gravitation. Hawking, S., W.; Israel, W. (eds.), Cambridge University Press. (1989).

[57] Ford, L. H. The Classical Singularity Theorems and Their Quantum Loopholes. Int. J. Theor. Phys. 42. (2003). 1219-1225.

[58] Penrose, R. Gravitational Collapse and Space-Time Singularities. Phys. Rev. Lett. 14. (1965). 57-64.

[59] Retter, A.; Heller, S. The revival of white holes as Small Bangs. New Astronomy 17. (2012). 73-75.

[60] Frolov, V. P.; Novikov, I. D. Black Hole Physics: Basic Concepts and New Developments. Springer (1998). pp. 580-581. 